\title{
Ad-Hoc Reviewers from 2015
}

(C) Springer Science+Business Media New York 2015

The Journal of Nonverbal Behavior greatly appreciates the assistance of the Editorial Board as well as the following ad hoc manuscript reviewers:

\begin{tabular}{|c|c|}
\hline Susan Andrzejewski & Jari Hietanen \\
\hline Anthony Atkinson & Sara Hodges \\
\hline Cord Benecke & Terrence Horgan \\
\hline Dario Bombari & Susan M. Hughes \\
\hline R. Thomas Boone & Keiki Ishii \\
\hline Manuel Calvo & Rachel Jack \\
\hline Elena Canadas & Lucy Johnston \\
\hline Dana R. Carney & Susanne Kaiser \\
\hline Mark Cary & Spencer D. Kelly \\
\hline Vanessa L. Castro & Peggy Kern \\
\hline Tara M. Chaplin & Sebastian Korb \\
\hline Mark Coulson & Tera Letzring \\
\hline Nele Dael & Timothy Levine \\
\hline Jonas Dietrich & Scott Lilienfield \\
\hline Katherine Duggan & Cesar Lima \\
\hline Daniel Dukes & Ottmar Lipp \\
\hline Heiner Ellgring & Kimberly Livingstone \\
\hline Sally Farley & Janek Lobmaier \\
\hline Marina Fiori & Lars-Olav Lundqvist \\
\hline Agneta Fischer & Takahiko Masuda \\
\hline Katrina Fong & Gary McKeown \\
\hline Wolfgang Friedlmeier & Marc Mehu \\
\hline Kornelia Gentsch & Christian Meissner \\
\hline Steven Gillespie & Alissa Melinger \\
\hline Pierre Gosselin & Lynden Miles \\
\hline Sarah D. Gunnery & Eric J. Moody \\
\hline Amy G. Halberstadt & Beth Morling \\
\hline Shlomo Hareli & Marcello Mortillaro \\
\hline Michael Haselhuhn & Laura Naumann \\
\hline Skylar Hawk & Paula Neidenthal \\
\hline Pa Her & Lauri Nummenmaa \\
\hline
\end{tabular}

Alison Parker

Alexandra Paxton

David Ian Perrett

K. V. Petrides

Robert R. Provine

Anne Richards

Helen Rodger

Katharina Rohlfing

Nicholas Rule

Magdalena Rychlowska

Katje Schlegal

Louis Tassinary

Theresa Taylor-Patridge

Pascal Thibault

Konstantin Tskhay

M. A. Tuk

Tanya Vacharkulksemsuk

Michel Valstar

Yolanda van Beek

Robrecht van der Wel

Edith Van Dyck

Lyn Van Swol

Ishabel Vicaria

Abbie Wazlawek

Lisa Welling

Cindy White

Matthias Weiser

Piotr Winkielman

Jolie Wormwood 